\title{
Review of: "Post-encoding modulation of spatial memory by a GABAA-agonist"
}

antonella lopez ${ }^{1}$

1 University of Bari

Potential competing interests: The author(s) declared that no potential competing interests exist.

The paper is interesting and and it is well written. Anyway, I have some suggestions to improve the manuscript.

The authors could better clarify the enrolment procedure. It seems not completely clear to me the procedure adopted for the control group. Moreover, how did the authors define the sample size?

Some parts of the discussion section could be moved to the introduction, so as to focus the discussion on the results. Moreover I suggest to add the limitations of the study and the future research directions.

I look forward to reviewing the final paper 